\title{
shRNA-mediated knockdown of KNTC1 suppresses cell viability and induces apoptosis in esophageal squamous cell carcinoma
}

\author{
CHUN-TAO LIU, LI MIN, YONG-JUN WANG, PENG LI, YONG-DONG WU and SHU-TIAN ZHANG
}

\author{
Department of Gastroenterology, Beijing Friendship Hospital, Capital Medical University, \\ National Clinical Research Center for Digestive Diseases, Beijing Digestive Disease Center, Beijing 100050, P.R. China
}

Received November 20, 2017; Accepted November 6, 2018

DOI: $10.3892 /$ ijo.2019.4672

\begin{abstract}
Kinetochore-associated proteins are critical components of mitotic checkpoints, which are essential for faithful chromosomal segregation and spindle assembly during cell division. Recent advances have demonstrated that kinetochore-associated proteins are upregulated and serve significant roles in the carcinogenesis of numerous types of cancer. However, the effects of kinetochore-associated protein 1 (KNTC1) on human cancer, particularly on esophageal squamous cell carcinoma (ESCC), remain unclear. The present study revealed that KNTC1 was highly expressed in ESCC cell lines. Subsequently, lentivirus-mediated short hairpin RNAs were used to knockdown KNTC1 expression in human ESCC cell lines. Cell growth and viability were measured using multiparametric high-content screening and the MTT assay, respectively. Cell apoptosis was assessed by staining cells with Annexin V-allophycocyanin and was detected using FACScan flow cytometry. The results demonstrated that knockdown of KNTC1 effectively inhibited cell viability and increased apoptosis. In addition, a gene set enrichment analysis of online ESCC datasets indicated that KNTC1 overexpression was associated with increases in the mitotic spindle and hypoxia pathways, and decreases in the DNA repair and mismatch repair pathways. The findings of the present study suggested that KNTC1 may have an essential role in mediating cell viability and apoptosis in human ESCC cells and may serve as a novel therapeutic target for ESCC.
\end{abstract}

\section{Introduction}

Esophageal cancer is the eighth most common malignancy and the sixth most common cause of cancer-associated mortality

Correspondence to: Professor Shu-Tian Zhang, Department of Gastroenterology, Beijing Friendship Hospital, Capital Medical University, National Clinical Research Center for Digestive Diseases, Beijing Digestive Disease Center, 95 Yong An Road, Beijing 100050, P.R. China

E-mail:drzstbj@126.com

Key words: apoptosis, esophageal squamous cell carcinoma, kinetochore-associated proteins, mitosis, viability worldwide (1). In the Chinese population, esophageal squamous cell carcinoma (ESCC) is the predominant histological subtype, which is responsible for $\sim 210,000$ cases of mortality each year, or $52 \%$ of all cases of ESCC-associated mortality worldwide (2). The characteristics of ESCC include being prone to invasive local growth, early lymphatic spread and vascular invasion $(3,4)$. ESCC has a very poor survival rate, despite significant progress in multimodal therapy. Numerous genetic alterations involved in the carcinogenesis of ESCCs have been reported (5); however, the exact molecular mechanisms remain unclear. Characterizing the precise mechanisms of esophageal carcinogenesis may help to identify biomarkers that can improve early diagnosis and targeted therapy of ESCC.

Kinetochore-associated protein 1 (KNTC1) is a key component of mitotic checkpoints, which ensures proper chromosomal segregation during cell division (6). Both chromosomal segregation and cell division are critical, highly ordered biological processes that are dependent on numerous evolutionarily conserved protein complexes. Many of the proteins that modulate the process of mitosis are overexpressed in human malignancies compared with their normal counterparts, and some have been reported to serve as oncogenes (7). KNTC2 is known to serve a critical role in chromosomal segregation during $\mathrm{M}$ phase (8). KNTC2 is upregulated in the tumor tissues of patients with various types of cancer, including gastric cancer, colorectal cancer, pancreatic cancer, hepatocellular carcinoma (HCC), breast cancer and non-small cell lung carcinoma (9-14). In addition, small interfering RNA (siRNA)-mediated silencing of KNTC2 has been reported to suppress cell proliferation and to induce apoptotic cell death in these tumor cells $(9,12)$. Furthermore, repeated administration of KNTC2 siRNAs has been revealed to specifically inhibit tumor growth in an orthotopic transplantation tumor model of HCC in nude mice (15). Targeted knockdown of kinetochore scaffold 1, which is widely expressed in various types of primary cancer, induces apoptosis in human transformed or tumor cell lines in vitro, and markedly impedes the growth of implanted tumors in vivo (16). These studies demonstrated that kinetochore proteins may serve as potential biomarkers for the early diagnosis of cancer, and the characterization of the role of kinetochore proteins may further contribute to the development of novel personalized treatments for human malignancies. 
KNTC1 is an evolutionarily conserved subunit of the kinetochore protein complex, which is vital for spindle assembly and chromosomal segregation. It elicits an inhibitory signal to prevent the onset of anaphase, until all chromosomes have been properly arranged in the spindle. Cells produce lagging chromosomes and chromatin bridges after being injected with KNTC1-blocking antibody, thus suggesting that KNTC1 is a vital component of the mitotic checkpoint (6). However, to the best of our knowledge, there are no previous reports describing the significance of KNTC1 in human malignancies, or in ESCC in particular.

The present study analyzed the expression levels of KNTC1 in ESCC cell lines. Subsequently, lentivirus-mediated short hairpin RNAs (shRNAs) were employed to investigate the effects of KNTC1 knockdown on human ESCC cell viability and apoptosis in vitro. In addition, gene set enrichment analysis (GSEA) was used to infer the possible mechanisms underlying the involvement of KNTC1 in ESCC tumorigenesis.

\section{Materials and methods}

Cell culture and human tissues. The human ESCC cell lines Eca-109, EC9706 and TE-1 were provided by the Cancer Institute and Hospital, Chinese Academy of Medical Sciences (Beijing, China). Authentication of cell lines was performed using short tandem repeat analysis. The three ESCC cell lines were cultured in RPMI-1640 medium (Invitrogen; Thermo Fisher Scientific, Inc., Waltham, MA, USA) supplemented with $10 \%$ fetal bovine serum (HyClone; GE Healthcare Life Sciences, Logan, UT, USA) and antibiotics (100 U/ml penicillin $\mathrm{G}$ and $100 \mu \mathrm{g} / \mathrm{ml}$ streptomycin) at $37^{\circ} \mathrm{C}$ and $95 \%$ humidity, in an atmosphere containing $5 \% \mathrm{CO}_{2}$. Normal esophageal tissues were obtained via endoscopic biopsy from two healthy volunteers (one male, 37 years old; one female, 44 years old; recruited on October 21,2017). Informed consent was obtained from the volunteers. The present study was approved by the ethics committees of the Capital Medical University Affiliated Beijing Friendship Hospital (Beijing, China).

$R N A$ extraction and reverse transcription-quantitative polymerase chain reaction $(R T-q P C R)$. Cells were pelleted using a cell scraper. Total RNA was isolated from cell pellets using TRIzol $^{\circledR}$ (Invitrogen; Thermo Fisher Scientific, Inc.), according to the manufacturer's protocol. Total RNA concentration and purity were measured with a UV spectrophotometer (Ultrospec 2100 Pro; GE Healthcare, Chicago, IL, USA). RT was performed using the PrimeScript RT reagent kit (Takara Biotechnology Co., Ltd., Dalian, China), according to the manufacturer's protocol. qPCR was performed using SYBR ${ }^{\circledR}$ Premix Ex Taq ${ }^{\mathrm{TM}}$ II (Takara Biotechnology Co., Ltd.) and the CFX96 $^{\mathrm{TM}}$ Real-Time system (Bio-Rad Laboratories, Inc., Hercules, CA, USA), according to the manufacturers' protocols. The specific primers used were as follows: KNTC1, forward 5'-GCAACAACTTGTAGACGACGCT-3', reverse 5'-TCAATCCAAGAACTGCCACTG-3'; and GAPDH, forward 5'-TGACTTCAACAGCGACACCCA-3' and reverse 5'-CACCCTGTTGCTGTAGCCAAA-3'. RT-qPCR consisted of an initial denaturation step at $95^{\circ} \mathrm{C}$ for $15 \mathrm{sec}$, followed by 45 cycles at $95^{\circ} \mathrm{C}$ for $5 \mathrm{sec}$ and $60^{\circ} \mathrm{C}$ for $30 \mathrm{sec}$. PCR reactions were optimized for the number of cycles to ensure product intensity within the logarithmic phase of amplification. The relative mRNA expression levels of KNTC1 were normalized to the GAPDH internal control. The relative mRNA expression levels were calculated using the $2^{-\Delta \Delta \mathrm{Cq}}$ method (17). The PCR products of KNTC1 and GAPDH were 270 and $121 \mathrm{bp}$, respectively.

Recombinant lentivirus vector production and transduction. The KNTC1-targeted short hairpin RNA (shRNA) sequence (5'-TGAGTTTATGGGATATTTA-3') was designed by Shanghai GeneChem Co. Ltd. (Shanghai, China). The shRNA sequences were synthesized and subsequently cloned into the pGCSIL-green fluorescent protein (GFP) lentiviral vector by Shanghai GeneChem Co. Ltd. The nonsense shRNA (5'-TTC TCCGAACGTGTCACGT-3'), which shared no homology with any known human genes, was generated as a negative control (NC) (Shanghai GeneChem Co. Ltd.). For recombinant lentiviral transduction, Eca-109 and TE-1 cells were seeded into 6-well plates until cell confluence reached $70 \%$, and were then infected with the KNTC1-shRNA-lentivirus or the NC lentivirus at a multiplicity of infection of 50. Transduction effects were determined by detecting GFP expression $24-72 \mathrm{~h}$ post-transduction using fluorescence and light microscopy. Cells were harvested $72 \mathrm{~h}$ post-transduction, and the knockdown effect was confirmed with RT-qPCR and western blot analysis.

Western blot analysis. Total protein was isolated from cells using the total protein extraction kit (Nanjing Keygen Biotech Co., Ltd., Nanjing, China), according to the manufacturer's protocol. Briefly, the cells were collected and were lysed in lysis buffer containing $150 \mathrm{mM}$ sodium chloride, $0.1 \mathrm{M}$ Tris, $1 \%$ (v/v) Tween-20, $50 \mathrm{mM}$ diethyldithiocarbamic acid, $1 \mathrm{mM}$ ethylenediaminetetraacetic acid and protease inhibitors at $\mathrm{pH}$ 8.0. The lysates were kept on ice for $30 \mathrm{~min}$ and were centrifuged at $12,000 \mathrm{x} \mathrm{g}$ for $15 \mathrm{~min}$ at $4^{\circ} \mathrm{C}$, and the supernatants were collected. Protein concentrations were measured using a Pierce bicinchoninic acid protein assay kit (Pierce; Thermo Fisher Scientific, Inc.). The protein samples (50 $\mu \mathrm{g} /$ lane) were separated by $10 \%$ SDS-PAGE according to Laemmli's method (18) and were then electrophoretically transferred to polyvinylidene fluoride membranes (EMD Millipore, Billerica, MA, USA). The membranes were blocked with 5\% non-fat dry milk in Tris-buffered saline containing $0.1 \%$ Tween-20 (TBST) buffer for $2 \mathrm{~h}$ at room temperature, followed by incubation with primary antibodies at a dilution of 1:2,000 at $4^{\circ} \mathrm{C}$ overnight. After being washed with TBST, the membranes were incubated with horseradish peroxidase-conjugated immunoglobulin $\mathrm{G}(\mathrm{IgG})$ secondary antibody (anti-rabbit IgG, cat. no. sc-2004 and anti-mouse IgG, cat. no. sc-2005; dilution 1:5,000; Santa Cruz Biotechnology, Inc. Dallas, TX, USA) for $1 \mathrm{~h}$ at room temperature. Immunoblots were developed using an enhanced chemiluminescence (ECL) reagent (ECL Plus; GE Healthcare). KNTC1 antibody (cat. no. ab85996) was purchased from Abcam (Cambridge, MA, USA) and GAPDH antibody (cat. no. sc-32233) was purchased from Santa Cruz Biotechnology, Inc.

Cell growth and viability assays. Cell growth was measured using multiparametric high-content screening (HCS), 
according to a previously reported method (19). Briefly, following lentiviral transduction, the cells were seeded at 2,000 cells/well in 96-well plates and were incubated at $37^{\circ} \mathrm{C}$ in an atmosphere containing $5 \% \mathrm{CO}_{2}$ for 5 days. The 96-well plates were scanned using Celigo Image Cytometer (version 4.1.3.0; Nexcelom Bioscience LLC, Lawrence, MA, USA) each day. The system includes a computerized fluorescence-imaging microscope that automatically identifies stained cells, and reports the intensity and distribution of the stained cells in each well. Fluorescence images were acquired for each channel (magnification, x20) using suitable filters.

Cell viability was measured using the MTT assay post-lentiviral transduction. Briefly, cells in the logarithmic growth phase were seeded in 96-well plates at a density of $1.0 \times 10^{4}$ cells $/ \mathrm{ml}$ in $200 \mu 1$ medium and were incubated at $37^{\circ} \mathrm{C}$ in an atmosphere containing $5 \% \mathrm{CO} 2$. Subsequently, the medium was replaced with serum-free RPMI-1640 containing $0.2 \mathrm{mg} / \mathrm{ml} \mathrm{MTT}$ at 24, 48, 72, 96 and $120 \mathrm{~h}$, and cells were incubated for an additional $4 \mathrm{~h}$ at $37^{\circ} \mathrm{C}$. After removal of the supernatant, $150 \mu \mathrm{l}$ dimethyl sulfoxide was added to each well, in order to completely dissolve the formazan. The plates were lightly vortexed and agitated for $10 \mathrm{~min}$, and optical density values were detected at $490 \mathrm{~nm}$ using a microplate reader (Bio-Rad Laboratories, Inc.).

Analysis of cell apoptosis and caspase-3/7 activities. Cell apoptosis was assessed by staining cells with Annexin V-allophycocyanin (APC) (eBioscience; Thermo Fisher Scientific, Inc.) and was detected using FACScan flow cytometry (FCM; BD Biosciences, Franklin Lakes, NJ, USA). Briefly, cells were plated in 6-well plates at a density of $2.0 \times 10^{4}$ cells $/ \mathrm{ml}$ in $3 \mathrm{ml}$ medium. After 5 days of transduction with the KNTC1-shRNA-lentivirus or NC-shRNA-lentivirus, the cells were harvested and washed twice with ice-cold PBS. The cells were then resuspended and adjusted to $1 \times 10^{6} / \mathrm{ml}$ with $1 \mathrm{X}$ staining buffer. A $100-\mu 1$ cell suspension was stained with $5 \mu \mathrm{l}$ Annexin V-APC for $15 \mathrm{~min}$ at room temperature in the dark and cell apoptosis was detected using FCM (Guava ${ }^{\circledR}$ easyCyte HT; EMD Millipore) within $1 \mathrm{~h}$.

Caspase-3 and caspase-7 activities of Eca-109 and TE-1 cells following KNTC1-shRNA-lentiviral transduction were detected using the Caspase-Glo ${ }^{\circledR}$ 3/7 assay (Promega Corporation, Madison, WI, USA), according to a previously reported method (20). Briefly, $72 \mathrm{~h}$ after lentiviral transduction, cells were seeded in 96-well microplates (Promega Corporation) at 10,000 cells/well. Briefly, the Caspase-Glo ${ }^{\circledR}$ $3 / 7$ reagent $(100 \mu \mathrm{l} /$ well) was added to each well, and the plates were gently agitated for $2 \mathrm{~h}$ at $20-25^{\circ} \mathrm{C}$. Finally, the luminescence was detected using a plate-reading luminometer.

GSEA. An online ESCC dataset with supplementary clinical information was downloaded from The Cancer Genome Atlas (TCGA) and was used for GSEA. The dataset contained mRNA profiling data for 97 patients with ESCC from TCGA and was downloaded from the API of cBioPortal (http://www. cbioportal.org/webAPI). A Pearson correlation analysis was performed to examine gene-gene expression correlations. Hierarchical clustering was performed to distinguish different subgroups, according to the expression levels of the given genes. Four gene sets were downloaded to perform the GSEA

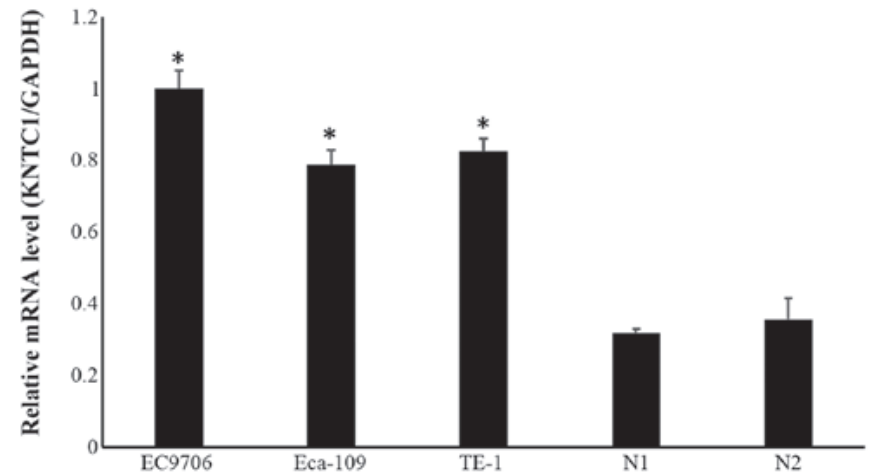

Figure 1. mRNA expression levels of KNTC1 in ESCC cell lines and normal esophageal tissues. KNTC1 mRNA expression was examined using reverse transcription-quantitative polymerase chain reaction. N1 and N2 refer to normal esophageal tissues. KNTC1 expression was significantly higher in ESCC cell lines compared with in normal esophageal tissues. ${ }^{*} \mathrm{P}<0.05$ vs. normal esophageal tissues. ESCC, esophageal squamous cell carcinoma; $\mathrm{KNTC1,} \mathrm{kinetochore-associated} \mathrm{protein} 1$.

analysis, including mitotic spindle, hypoxia, DNA repair and mismatch repair, which were located in the Molecular Signatures Database (MSigDB) and the Kyoto Encyclopedia of Genes and Genomes (KEGG), respectively $(21,22)$. The GSEA was performed to evaluate the correlation between KNTC1 expression and these four gene sets.

Statistical analysis. All of the results are presented as the mean values of at least three independent experiments using cells from separate cultures. Data are presented as the means \pm standard deviation. Differences between two groups were analyzed using Student's t-test. For comparisons of more than two groups, repeated measures analysis of variance, followed by Tukey's multiple comparison test, was conducted. $\mathrm{P}<0.05$ was considered to indicate a statistically significant difference. Statistical analyses were performed using SPSS standard version 16.0 (SPSS, Inc., Chicago, IL, USA) and R 3.1.2 software (www.r-project.org).

\section{Results}

Expression levels of KNTC1 in ESCC cell lines and normal esophageal tissues. The mRNA expression levels of KNTC1 were assessed in the ESCC cell lines Eca-109, EC9706 and TE-1, and in two normal esophageal tissues using RT-qPCR. The results revealed that KNTC1 expression was significantly higher in ESCC cell lines compared with in normal esophageal tissues (Fig. 1).

Lentivirus-mediated shRNA specifically inhibits KNTC1 expression. To explore the role of KNTC1 knockdown in the biological behaviors of ESCC cells, a lentivirus-mediated shRNA that targeted KNTC1 was introduced into Eca-109 and TE-1 cells, both of which expressed high levels of KNTC1. A fluorescence microscope was used to determine the transduction efficacy of the recombinant lentivirus. Transduction efficiencies reached $80 \%$ in both cell lines. As shown in Fig. 2, by day 3 post-transduction with KNTC1-shRNA or NC-shRNA lentiviruses, the proportion of infected cells was $>80 \%$. Subsequently, the knockdown efficacy of KNTC1-shRNA was 


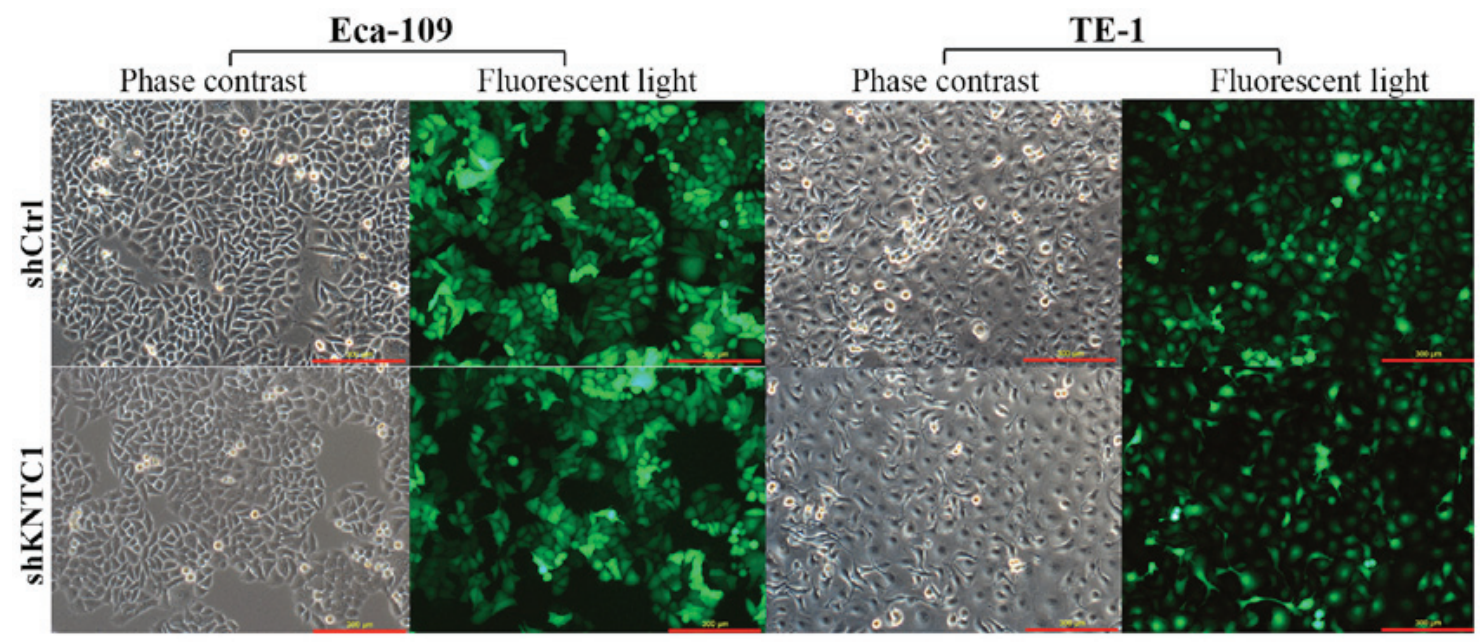

Figure 2. Assessing the transduction efficiency of shKNTC1 or shCtrl lentivirus vectors in Eca-109 and TE-1 cells. Eca-109 and TE-1 cells were infected with shKNTC1 or shCtrl and were examined by fluorescence and light microscopy at day 3 post-transduction. Magnification, x100. Representative images of the cultures are shown. Ctrl, control; KNTC1, kinetochore-associated protein 1; sh, short hairpin RNA.

A

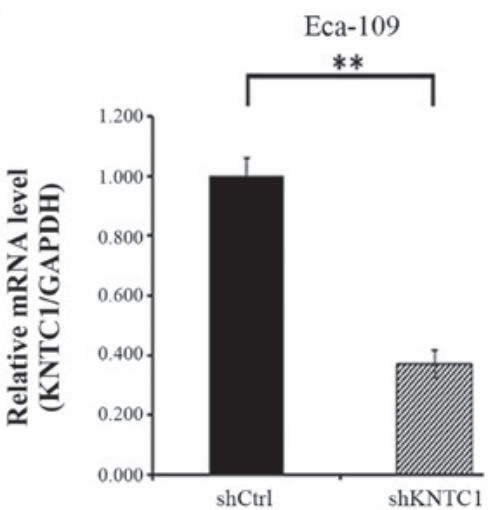

B

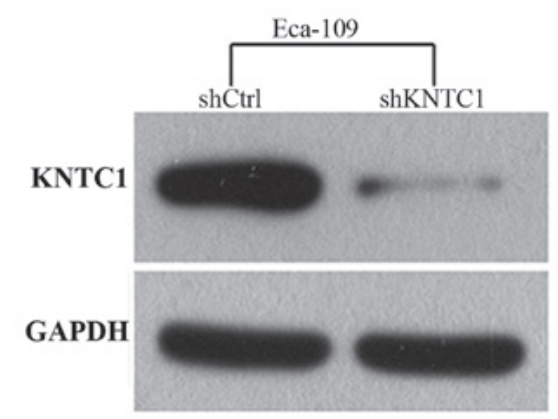

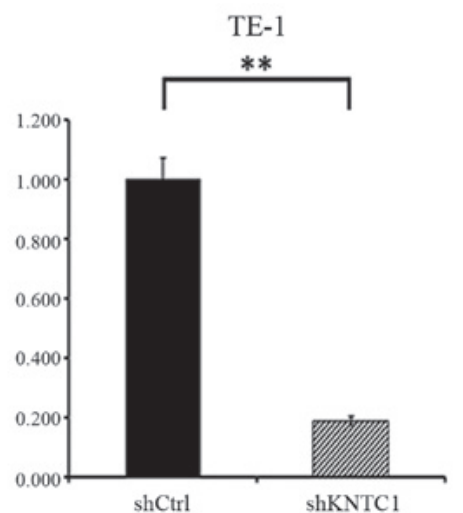

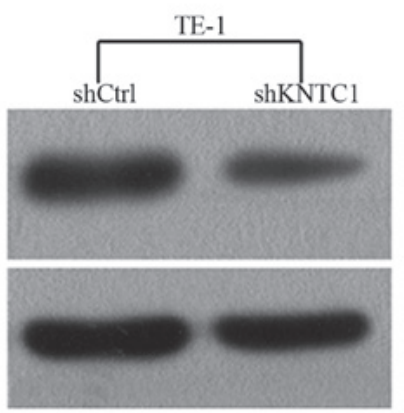

Figure 3. Confirmation of KNTC1 knockdown in Eca-109 and TE-1 cells, as determined by reverse transcription-quantitative polymerase chain reaction and western blotting. (A) mRNA expression levels of KNTC1 in Eca-109 and TE-1 cells transduced with shKNTC1 were decreased by $~ 63$ and $81 \%$, respectively, compared with in cells transduced with shCtrl after $72 \mathrm{~h}$. ${ }^{* *} \mathrm{P}<0.01$. (B) Protein expression levels of KNTC1 were significantly decreased following KNTC1 knockdown in Eca-109 and TE-1 cells. Ctrl, control; KNTC1, kinetochore-associated protein 1; sh, short hairpin RNA.

confirmed by RT-qPCR and western blotting. As shown in Fig. 3A, the mRNA expression levels of KNTC1 in Eca-109 and TE-1 cells transduced with the KNTC1-shRNA lentivirus were decreased by $\sim 63$ and $81 \%$, respectively, compared with in the cells transduced with the NC-shRNA lentivirus after $72 \mathrm{~h}(\mathrm{P}<0.01)$. KNTC1 protein expression was also examined by western blotting in these cells; the protein expression levels of KNTC1 were significantly reduced in KNTC1-shRNA lentivirus-transfected cells, thus indicating the effective knockdown of KNTC1 (Fig. 3B).
Knockdown of KNTC1 inhibits growth and viability of ESCC cells. HCS was performed to assess the effects of KNTC1 knockdown on ESCC cell growth in vitro. Eca-109 and TE-1 cells expressing either KNTC1-shRNA or NC-shRNA lentiviruses were plated in 96-well plates and were assessed using HCS every day for 5 days. Cell growth rate was defined as the ratio of the cell count on a certain day to the cell count on the first day. As illustrated in Fig. 4A, the growth rates of Eca-109 cells transduced with the KNTC1-shRNA lentivirus were significantly reduced compared with in the cells transduced with the NC-shRNA 
A
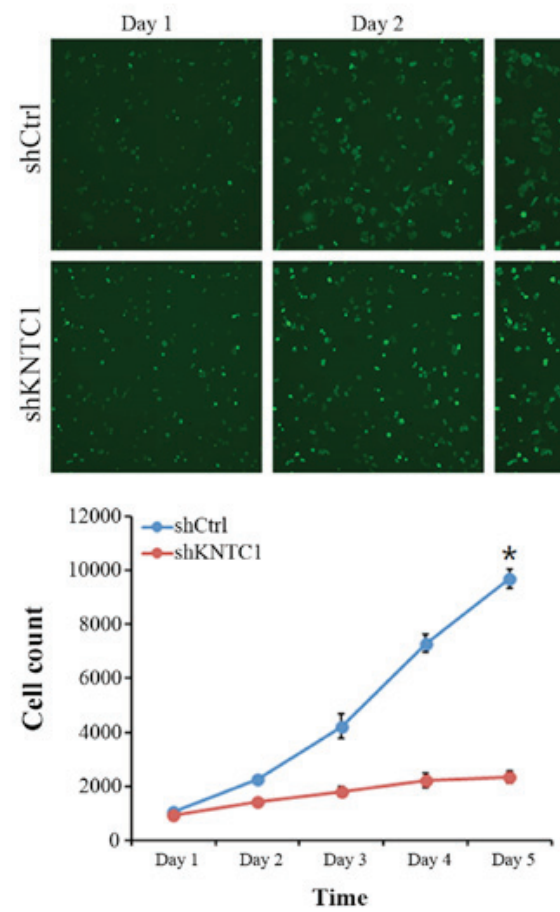

B

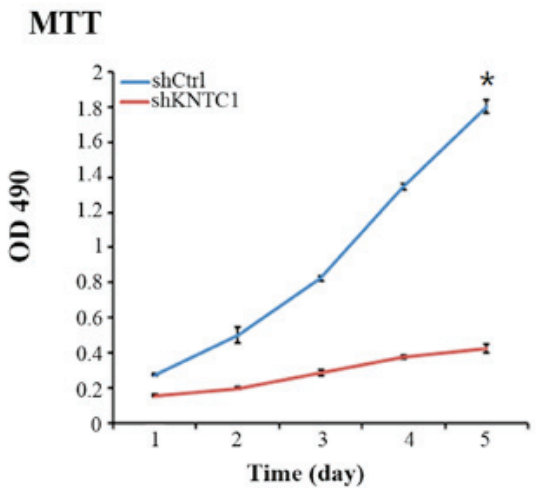

Eca-109
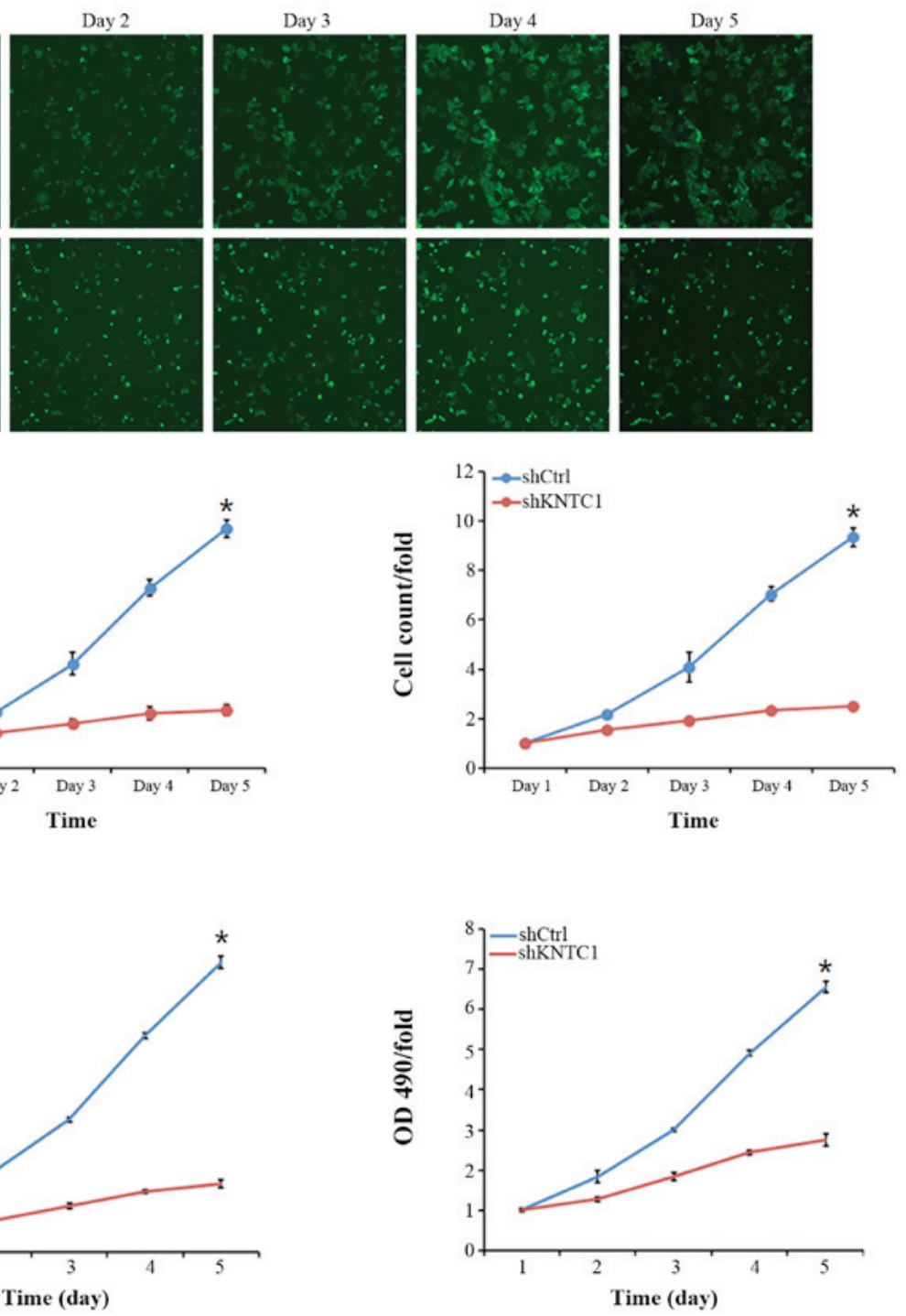

Figure 4. Knockdown of KNTC1 suppresses growth and viability of Eca-109 cells. Cell growth and viability were determined by high-content screening and MTT assays every day for 5 days. (A) High-content cell imaging was performed every day to acquire images of cell growth (magnification, x20). Cell growth rates of the Eca-109 cells transduced with shKNTC1 were significantly reduced compared with those transduced with shCtrl (P<0.01). Cell Count/Fold refers to fold change in cell count compared with at day 1. Staining marker, green fluorescent protein. (B) Similar results were also observed using the MTT assay. OD490/Fold refers to fold change in OD490 compared with at day 1. "P<0.01. Ctrl, control; KNTC1, kinetochore-associated protein 1; OD, optical density; sh, short hairpin RNA.

lentivirus $(\mathrm{P}<0.01)$. As shown in Fig. 4B, similar results were observed using the MTT assay, which further corroborated the negative effect of KNTC1 knockdown on Eca-109 cell viability $(\mathrm{P}<0.01)$. Taken together, these results suggested that KNTC1 knockdown significantly inhibited the viability of Eca-109 and TE-1 cells, thus indicating that KNTC1 may serve a critical role in the growth and viability of ESCC cells.

Knockdown of KNTC1 in ESCC cells increases cell apoptosis. To investigate whether KNTC1 expression affects apoptotic cell death in ESCC cells, KNTC1 was knocked down in Eca-109 and TE-1 cells. Cell apoptosis was assessed with Annexin V staining and FCM. As shown in Fig. 5, cell apoptosis of Eca-109 was significantly increased in the KNTC1-shRNA lentivirus group compared with in the NC-shRNA lentivirus group $(\mathrm{P}<0.01)$. TE-1 cell line exhibited similar results (data not shown). To further confirm the ability of KNTC1 knockdown to induce apoptosis, caspase-3/7 activity analysis was performed. In accordance with the aforementioned observations, caspase-3/7 activity was markedly elevated in the KNTC1-shRNA lentivirus group compared with in the NC-shRNA lentivirus group $(\mathrm{P}<0.01)$. These results indicated that KNTC1 expression may be a determinant of cell apoptosis in Eca-109 and TE-1 cells.

Association between KNTC1 expression and mitotic spindle, hypoxia, DNA repair and mismatch repair gene sets. Four gene sets were downloaded to perform the GSEA, including the mitotic spindle, hypoxia, DNA repair and mismatch repair gene sets. The normalized enrichment scores (NES) of the mitotic spindle and hypoxia gene sets were $1.016(\mathrm{P}<0.001)$ and $1.004(\mathrm{P}=0.005)$, respectively (Fig. 6A), indicating that 


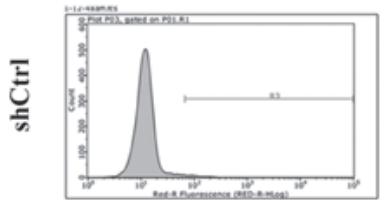

Annexin V

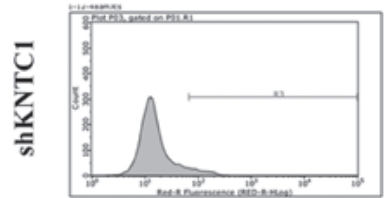

Annexin V

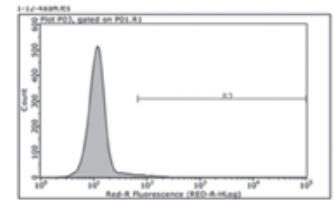

Annexin V

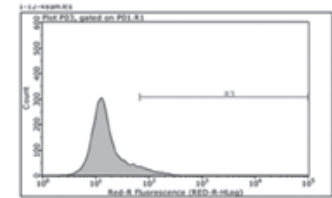

Annexin V

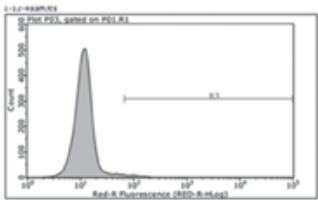

Annexin V

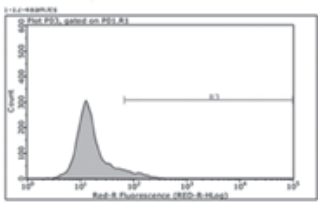

Annexin V
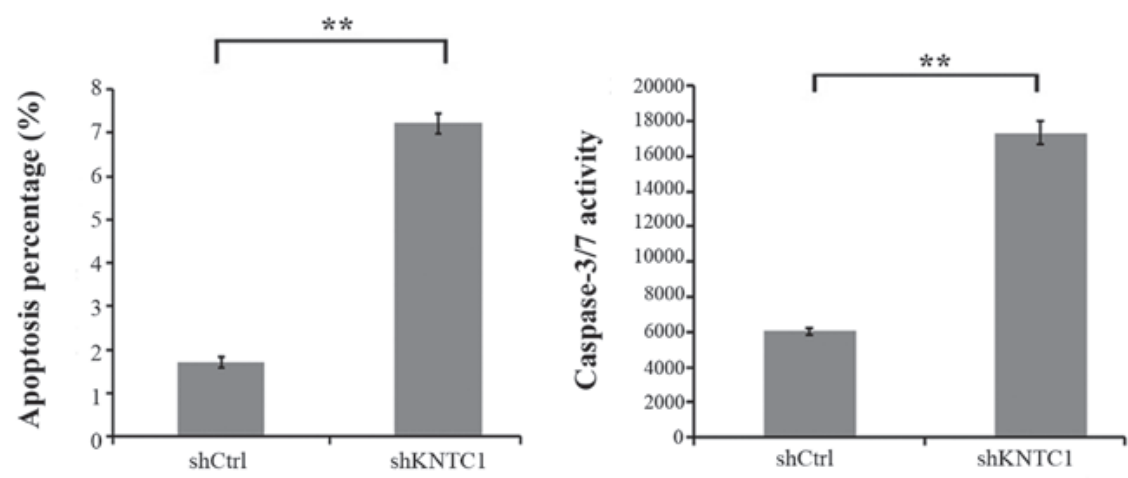

Figure 5. Knockdown of KNTC1 increases the apoptosis of Eca-109 cells. Cell apoptosis was assessed using Annexin V staining and flow cytometry. Cell apoptosis was significantly increased in the shKNTC1 lentivirus group (7.22 \pm 0.23$)$ compared with in the shCtrl group (1.71 \pm 0.12$)$. Caspase-3/7 activities of Eca-109 cells following shKNTC1 lentiviral transduction were evaluated. Caspase-3/7-activities were markedly elevated in the shKNTC1 lentivirus group $(17,330.7 \pm 641.7)$ compared with in the shCtrl group $(6,041.0 \pm 179.2)$. ${ }^{* *} \mathrm{P}<0.01$. Ctrl, control; KNTC1, kinetochore-associated protein 1 ; sh, short hairpin RNA.

A
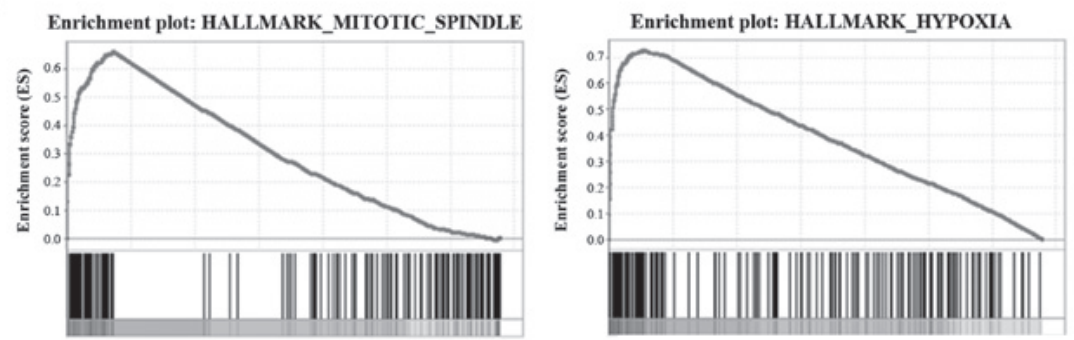

B
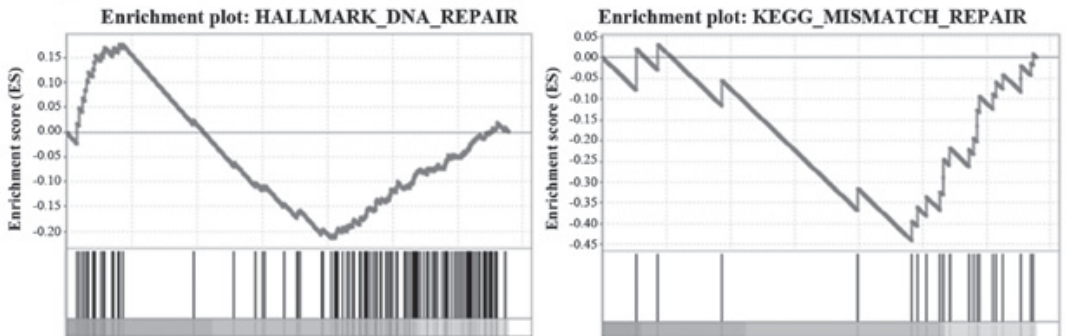

Figure 6. Gene set enrichment analysis using the online esophageal squamous cell carcinoma dataset from The Cancer Genome Atlas. (A) NES of the mitotic spindle and hypoxia gene sets were $1.016(\mathrm{P}<0.001)$ and $1.004(\mathrm{P}=0.005)$, respectively, indicating that $\mathrm{KNTC} 1$ expression was positively correlated with the mitotic spindle and hypoxia pathways. (B) For the DNA repair and mismatch repair gene sets, the NES were $-1.064(\mathrm{P}=0.021)$ and -1.093 ( $\mathrm{P}=0.015)$, respectively, indicating that KNTC1 expression was negatively correlated with the DNA repair and mismatch repair pathways. KNTC1, kinetochore-associated protein 1; NES, normalized enrichment scores.

KNTC1 expression may be positively correlated with the mitotic spindle and hypoxia pathways. For the DNA repair and mismatch repair gene sets, the NES were $-1.064(\mathrm{P}=0.021)$ and $-1.093(\mathrm{P}=0.015)$, respectively (Fig. 6B), indicating that KNTC1 expression was negatively correlated with the DNA repair and mismatch repair pathways.

\section{Discussion}

Proper and accurate chromosomal segregation is essential for maintaining genomic integrity during cell division. A properly functioning centromere and its associated kinetochore are crucial in determining the fidelity of chromosomal segregation 
during mitosis. This step is under the control of numerous proteins and protein complexes, which are localized to the kinetochores and monitor the accuracy of this process (23). Defects in centromere- and kinetochore-associated proteins have been proposed to contribute to aneuploidy and chromosomal instability, which are associated with cancer progression in several types of human tumor $(24,25)$. The presence of aneuploid cells is detected in $\sim 90 \%$ of solid tumors and $\sim 50 \%$ of hematopoietic cancers (26). Chromosomal instability is believed to be the driver of cancer predisposition, progression and intratumoral heterogeneity, whereas aneuploidy and chromosomal instability have been attributed to poor patient prognosis, metastasis and chemotherapeutic resistance (27).

In a previous study, upon analyzing the publicly available expression compendia of human normal tissues and cancer samples, a group of kinetochore genes, which encode proteins functioning exclusively at the kinetochores, has been revealed to be concurrently upregulated in cancers (28). Their upregulation coincides with the increased expression of genes associated with the cell cycle and DNA replication, thus suggesting a widespread activation of the cell division program potentially readying the cells for division whenever the signal for cell cycle entry is received (28). These kinetochore proteins have been noted for their involvement in increasing aneuploidy rates and the risk of tumor progression. KNTC1 is a core kinetochore protein that is required for faithful chromosomal segregation and spindle assembly. Experimental evidence has indicated that KNTC1 functions in a similar manner to that of the Drosophila rough deal protein. The three activities that have so far been ascribed to KNTC1 are as follows: i) Recruiting cytoplasmic dynein to the kinetochore; ii) participating in the poleward movement of chromosomes during mitosis; and iii) maintaining a functional metaphase checkpoint (29). However, to the best of our knowledge, no functional information is available to date regarding the function of $\mathrm{KNTC1}$ in human cancer, particularly in ESCC.

The present study examined the mRNA expression levels of KNTC1 in three ESCC cell lines and revealed that it was expressed in all tested cell lines. To explore the role of KNTC1 in ESCC, a KNTC1-shRNA lentiviral vector was constructed, which efficiently silenced KNTC1 in Eca-109 and TE-1 cell lines. HCS and MTT were performed to assess the effects of KNTC1 knockdown on ESCC cell growth and viability. Compared with the NC-shRNA lentivirus-transduced cells, KNTC1-shRNA-transduced cells exhibited significantly reduced cell growth and viability. In addition, knockdown of KNTC1 increased apoptosis of Eca-109 and TE-1 cells. Taken together, these results strongly suggested that KNTC1 may serve an oncogenic role in cell growth, viability and apoptosis in ESCC.

To further explore the mechanisms underlying KNTC1-mediated promotion of ESCC tumorigenesis, a GSEA was conducted using online ESCC datasets from TCGA. Three hallmark MSigDB gene sets and one KEGG gene set were adopted for analysis, including the mitotic spindle, hypoxia, DNA repair and mismatch repair gene sets. The results revealed that KNTC1 expression was positively correlated with the mitotic spindle and hypoxia gene sets, and negatively correlated with the DNA repair and mismatch repair gene sets. Hypoxia signaling regulates diverse biological processes, such as glycolysis, angiogenesis and invasion, and leads to genomic instability, promotion of cancer progression, increased metastatic potential, increased resistance to chemotherapy and radiation, and poor prognosis (30). A previous study revealed that hypoxia contributes to the metastasis and angiogenesis of ESCC (31). Hypoxia is known to induce phosphorylation of histone $\mathrm{H} 2 \mathrm{AX}$, which not only serves as a DNA damage marker but also has a critical role in mediating homologous DNA repair (32). Moderate hypoxia can lead to replication stress and activation of the DNA damage repair pathway proteins (33). Conversely, hypoxia-reoxygenation induces DNA damage through reactive oxygen species (34). DNA mismatch repair (MMR) is a highly conserved biological pathway that has a critical role in maintaining the fidelity of DNA replication, mutation avoidance and genomic stability. MMR also suppresses homologous recombination and was recently reported to serve a role in DNA damage signaling. Defects in MMR are associated with genome-wide instability, predisposition to several types of human cancer, including ESCC, and resistance to certain chemotherapeutic agents $(35,36)$. The cellular response to DNA damage is to limit viability, whilst initiating DNA repair. The present GSEA results detected increases in the mitotic spindle and hypoxia gene sets, and decreases in the DNA repair and mismatch repair gene sets in response to KNTC1 overexpression in ESCC. Therefore, it was inferred that KNTC1 overexpression may induce genomic instability and DNA damage through increased mitotic spindle and hypoxia pathways, which cannot be corrected by the decreased DNA repair and mismatch repair pathways, which may eventually lead to carcinogenesis and development of ESCC. However, the key molecular mechanisms underlying the malignant functions of KNTC1 require further investigation. We aim to further explore the molecular mechanisms, particularly the effects of KNTC1 on hypoxia and DNA repair.

In conclusion, the present study confirmed that knockdown of KNTC1 expression by shRNA inhibited cell viability and induced cell apoptosis in ESCC cell lines. In addition, GSEA of online ESCC datasets revealed that KNTC1 overexpression was associated with increases in the mitotic spindle and hypoxia pathways, and decreases in the DNA repair and mismatch repair pathways. However, further studies are required to reveal the precise mechanism of KNTC1 in the carcinogenesis and development of ESCC.

\section{Acknowledgments}

Not applicable.

\section{Funding}

The present study was supported by the Beijing Municipal Administration of Hospitals Youth Programme (grant no. QML20150105) and the Beijing Excellent Talents Training Program (grant no. 2015000021469G233).

\section{Availability of data and materials}

The datasets used and/or analyzed during the current study are available from the corresponding author on reasonable request. 


\section{Authors' contributions}

CTL and STZ made substantial contributions to the conception and design of the study. CTL performed the majority of the study and wrote the manuscript. LM performed the GSEA. YJW, PL and YDW were involved in designing the experiments, analyzing the data and editing the manuscript. All authors read and approved the final draft of the manuscript.

\section{Ethics approval and consent to participate}

Informed consent was obtained from the volunteers. The present study was approved by the ethics committees of the Capital Medical University Affiliated Beijing Friendship Hospital.

\section{Patient consent for publication}

Informed consent was obtained from all participants.

\section{Competing interests}

The authors declare that they have no competing interests.

\section{References}

1. Ferlay J, Soerjomataram I, Dikshit R, Eser S, Mathers C Rebelo M, Parkin DM, Forman D and Bray F: Cancer incidence and mortality worldwide: Sources, methods and major patterns in GLOBOCAN 2012. Int J Cancer 136: E359-E386, 2015.

2. He YT, Li DJ, Liang D, Jin J, Wen DG, Chen WQ and He J: Estimated of esophageal cancer incidence and mortality in China 2013. Zhonghua Zhong Liu Za Zhi 39: 315-320, 2017 (In Chinese).

3. Enzinger PC and Mayer RJ: Esophageal cancer. N Engl J Med 349: 2241-2252, 2003

4. Li JY: Epidemiology of esophageal cancer in China. Natl Cancer Inst Monogr 62: 113-120, 1982.

5. Liu X,Zhang M, Ying S,Zhang C,Lin R,Zheng J,Zhang G, Tian D, Guo Y, Du C, et al: Genetic Alterations in Esophageal Tissues From Squamous Dysplasia to Carcinoma. Gastroenterology 153: 166-177, 2017.

6. Chan GK, Jablonski SA, Starr DA, Goldberg ML and Yen TJ: Human Zw10 and ROD are mitotic checkpoint proteins that bind to kinetochores. Nat Cell Biol 2: 944-947, 2000.

7. Keen $\mathrm{N}$ and Taylor S: Aurora-kinase inhibitors as anticancer agents. Nat Rev Cancer 4: 927-936, 2004.

8. Chen Y, Riley DJ, Chen PL and Lee WH: HEC, a novel nuclear protein rich in leucine heptad repeats specifically involved in mitosis. Mol Cell Biol 17: 6049-6056, 1997.

9. Kaneko N, Miura K, Gu Z, Karasawa H, Ohnuma S, Sasaki H, Tsukamoto N, Yokoyama S, Yamamura A, Nagase H, et al: siRNA-mediated knockdown against CDCA1 and KNTC2, both frequently overexpressed in colorectal and gastric cancers, suppresses cell proliferation and induces apoptosis. Biochem Biophys Res Commun 390: 1235-1240, 2009.

10. Qu Y, Li J, Cai Q and Liu B: Hec1/Ndc80 is overexpressed in human gastric cancer and regulates cell growth. J Gastroenterol 49: 408-418, 2014.

11. Bièche I, Vacher S, Lallemand F, Tozlu-Kara S, Bennani H, Beuzelin M, Driouch K, Rouleau E, Lerebours F, Ripoche H, et al Expression analysis of mitotic spindle checkpoint genes in breast carcinoma: Role of NDC80/HEC1 in early breast tumorigenicity, and a two-gene signature for aneuploidy. Mol Cancer 10: 23, 2011.

12. Hayama S, Daigo Y, Kato T, Ishikawa N, Yamabuki T, Miyamoto M, Ito T, Tsuchiya E, Kondo $\mathrm{S}$ and Nakamura Y: Activation of CDCA1-KNTC2, members of centromere protein complex, involved in pulmonary carcinogenesis. Cancer Res 66: 10339-10348, 2006.

13. Meng QC, Wang HC, Song ZL, Shan ZZ, Yuan Z, Zheng Q and Huang XY: Overexpression of NDC80 is correlated with prognosis of pancreatic cancer and regulates cell proliferation. Am J Cancer Res 5: 1730-1740, 2015.
14. Huang LY, Chang CC, Lee YS, Huang JJ, Chuang SH, Chang JM, Kao KJ, Lau GM, Tsai PY, Liu CW, et al: Inhibition of Hecl as a novel approach for treatment of primary liver cancer. Cancer Chemother Pharmacol 74: 511-520, 2014.

15. Makita Y, Murata S, Katou Y, Kikuchi K, Uejima H, Teratani M, Hoashi Y, Kenjo E, Matsumoto S, Nogami M, et al: Anti-tumor activity of KNTC2 siRNA in orthotopic tumor model mice of hepatocellular carcinoma. Biochem Biophys Res Commun 493: 800-806, 2017

16. Urata YN, Takeshita F, Tanaka H, Ochiya T and Takimoto M: Targeted Knockdown of the Kinetochore Protein D40/Knl-1 Inhibits Human Cancer in a p53 Status-Independent Manner. Sci Rep 5: 13676, 2015.

17. Livak KJ and Schmittgen TD: Analysis of relative gene expression data using real-time quantitative PCR and the 2(-Delta Delta C(T)) method. Methods 25: 402-408, 2001.

18. Laemmli UK: Cleavage of structural proteins during the assembly of the head of bacteriophage T4. Nature 227: 680-685, 1970.

19. Zhou Y, Su Z, Huang Y, Sun T, Chen S, Wu T, Chen G, Xie X, Li B and Du Z: The Zfx gene is expressed in human gliomas and is important in the proliferation and apoptosis of the human malignant glioma cell line U251. J Exp Clin Cancer Res 30: 114 , 2011.

20. Abe W, Nasu K, Nakada C, Kawano Y, Moriyama M and Narahara H: miR-196b targets c-myc and Bcl-2 expression, inhibits proliferation and induces apoptosis in endometriotic stromal cells. Hum Reprod 28: 750-761, 2013.

21. Liberzon A, Birger C, Thorvaldsdóttir H, Ghandi M, Mesirov JP and Tamayo P: The Molecular Signatures Database (MSigDB) hallmark gene set collection. Cell Syst 1: 417-425, 2015.

22. Du J, Yuan Z, Ma Z, Song J, Xie X and Chen Y: KEGG-PATH: Kyoto encyclopedia of genes and genomes-based pathway analysis using a path analysis model. Mol Biosyst 10: 2441-2447, 2014.

23. Cleveland DW, Mao Y and Sullivan KF: Centromeres and kinetochores: From epigenetics to mitotic checkpoint signaling. Cell 112: 407-421, 2003.

24. Orr B, Godek KM and Compton D: Aneuploidy. Curr Biol 25: R538-R542, 2015.

25. Hartwell LH and Kastan MB: Cell cycle control and cancer. Science 266: 1821-1828, 1994.

26. Beh TT and Kalitsis P: The Role of Centromere Defects in Cancer. Prog Mol Subcell Biol 56: 541-554, 2017.

27. Thompson SL and Compton DA: Chromosomes and cancer cells Chromosome Res 19: 433-444, 2011.

28. Thiru P, Kern DM, McKinley KL, Monda JK, Rago F, Su KC, Tsinman T, Yarar D, Bell GW and Cheeseman IM: Kinetochore genes are coordinately up-regulated in human tumors as part of a FoxM1-related cell division program. Mol Biol Cell 25: 1983-1994, 2014.

29. Scaërou F, Starr DA, Piano F, Papoulas O, Karess RE and Goldberg ML: The ZW10 and Rough Deal checkpoint proteins function together in a large, evolutionarily conserved complex targeted to the kinetochore. J Cell Sci 114: 3103-3114, 2001.

30. Wilson WR and Hay MP: Targeting hypoxia in cancer therapy. Nat Rev Cancer 11: 393-410, 2011.

31. Li Y, Fu L, Li JB, Qin Y, Zeng TT, Zhou J, Zeng ZL, Chen J, Cao TT, Ban X, et al: Increased expression of EIF5A2, via hypoxia or gene amplification, contributes to metastasis and angiogenesis of esophageal squamous cell carcinoma. Gastroenterology 146 : 1701-1713.e9, 2014.

32. Sulli G, Di Micco R and d'Adda di Fagagna F: Crosstalk between chromatin state and DNA damage response in cellular senescence and cancer. Nat Rev Cancer 12: 709-720, 2012

33. Olcina MM, Foskolou IP, Anbalagan S, Senra JM, Pires IM, Jiang Y, Ryan AJ and Hammond EM: Replication stress and chromatin context link ATM activation to a role in DNA replication. Mol Cell 52: 758-766, 2013.

34. Hammond EM, Dorie MJ and Giaccia AJ: ATR/ATM targets are phosphorylated by ATR in response to hypoxia and ATM in response to reoxygenation. J Biol Chem 278: 12207-12213, 2003.

35. Liu D, Keijzers G and Rasmussen LJ: DNA mismatch repair and its many roles in eukaryotic cells. Mutat Res 773: 174-187, 2017.

36. Ling ZQ, Li P, Ge MH, Hu FJ, Fang XH, Dong ZM and Mao WM: Aberrant methylation of different DNA repair genes demonstrates distinct prognostic value for esophageal cancer. Dig Dis Sci 56: 2992-3004, 2011 